\title{
Heterogeneous surface growth and gelation of cement hydrates
}

\author{
Abhay Goyal, ${ }^{\dagger}$ Katerina loannidou, ${ }^{\ddagger}, \mathbf{9}, \S$ Christopher Tiede, ${ }^{\dagger}$ Pierre Levitz, $\|$ \\ Roland J.-M. Pellenq, ${ }^{\boldsymbol{T}, \S, \dagger}$ and Emanuela Del Gado*,† \\ $\dagger$ Department of Physics, Institute for Soft Matter Synthesis and Metrology, Georgetown \\ University, Washington, D.C. 20057 \\ $\ddagger$ Laboratoire de Mécanique et Génie Civil, CNRS, Université de Montpellier, 34090 \\ Montpellier, France
}

\Massachusetts Institute of Technology/CNRS/Aix-Marseille University Joint Laboratory, Cambridge, MA 02139

$\S$ Department of Civil and Environmental Engineering, Massachusetts Institute of Technology, Cambridge, MA 02139

||Physico-Chimie des Électrolytes et Nanosystèmes Interfaciaux, PHENIX, Sorbonne Université, CNRS, F-75005 Paris, France

E-mail: ed610@georgetown.edu 


\section{Local Ordering}

To characterize the differences in the local ordering, we looked at the Steinhardt Bond Orientational Order Parameters (BOOPs). ${ }^{1}$ These are computed by looking at angular orientation of "bonds" (not chemical bonds, but rather bonds defined by a suitable nearest neighbor selection). For a bond with some orientation $\vec{r}$, we consider the spherical harmonic function, $Y_{l m}(\theta(\vec{r}) \phi(\vec{r}))$. The BOOPs, $q_{l m}$, are defined as an average over the bonds of a particle:

$$
q_{l m}(i)=\frac{1}{N_{b_{i}}} \sum_{j=1}^{N_{b_{i}}} Y_{l m}\left(\theta\left(\overrightarrow{r_{i j}}\right) \phi\left(\overrightarrow{r_{i j}}\right)\right)
$$

We are interested in the even $l$ terms, which are independent of an arbitrary choice of bond direction. These terms are still dependent on the choice of reference frame though. To remove this dependence, we must consider rotationally invariant combinations of $q_{l m}$. The second-order rotational invariants are

$$
q_{l}(i)=\sqrt{\frac{4 \pi}{2 l+1} \sum_{m=-l}^{l}\left|q_{l m}(i)\right|^{2}}
$$

and the third-order invariants are

$$
\hat{w}_{l}(i)=w_{l}(i) /\left[\sum_{m=-l}^{l}\left|q_{l m}(i)\right|^{2}\right]^{3 / 2}
$$

where

$$
w_{l}(i)=\sum_{m_{1}, m_{2}, m_{3}}\left(\begin{array}{ccc}
l & l & l \\
m_{1} & m_{2} & m_{3}
\end{array}\right) q_{l m_{1}}(i) q_{l m_{2}}(i) q_{l m_{3}}(i)
$$

where the coefficients are the Wigner 3-j coefficients and the sum is over values such that $m_{1}+m_{2}+m_{3}=0$. Specifically, the set of $\left\{q_{4}, q_{6}, \hat{w}_{4}, \hat{w}_{6}\right\}$ are generally sufficient to characterize the order of colloidal gels. Here, we focus on $\hat{w}_{6}$ because it captures the difference between crystalline (fcc and hcp) and Bernal Spiral ordering (which, as shown below, is very prevalent 
in certain conditions).

In Fig. S1 we see that both ES and LS at $\phi=20 \%$ have clear signals of Bernal spirals in the central region. At the edge, ES maintains that ordering while the denser LS shifts into a more crystalline morphology. This further demonstrates that LS is more sensitive to the heterogeneity introduced in our system. While the percolated ES system is quite uniform, with LS the percolation leads to increasing morphological heterogeneity.
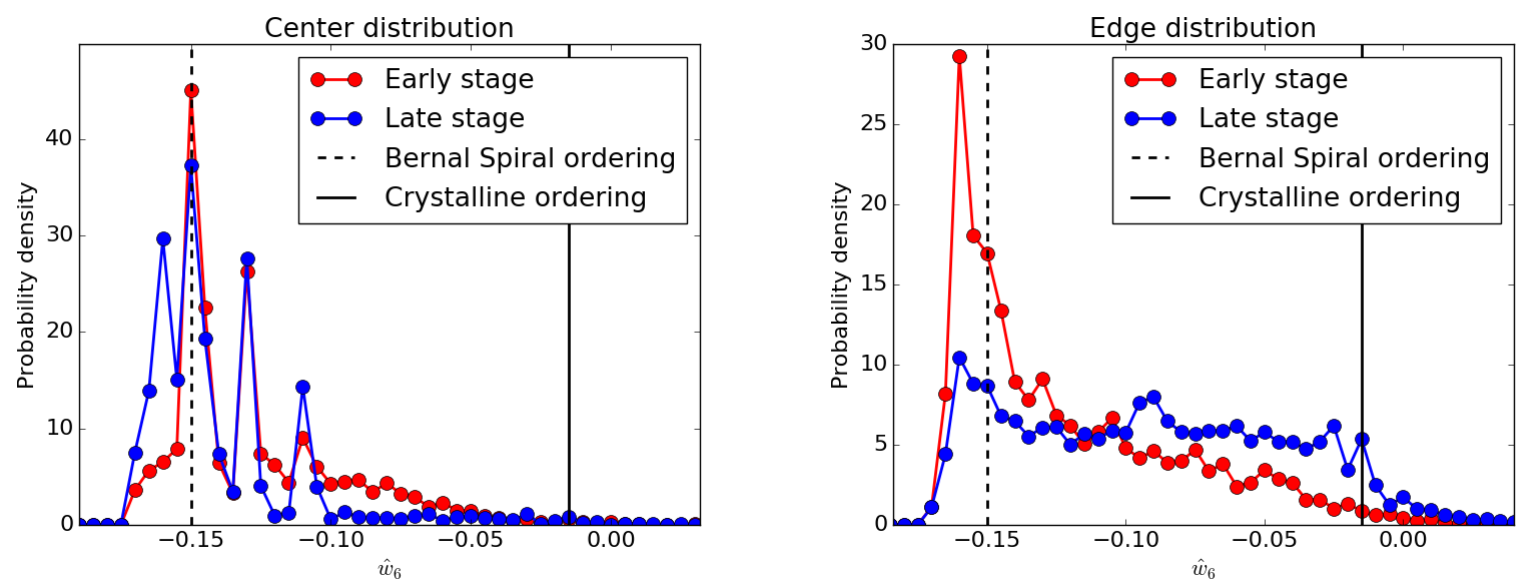

Figure S1: The distributions of the local BOOP as first introduced by Steinhardt. ${ }^{1}$ This provides a way to quantify the spatial differences in ordering. Here we show the rotational invariant $\hat{w}_{6}$ which is useful for differentiating between Bernal Spiral ordering and crystalline fcc/hcp ordering.

The concept of two different morphological forms of C-S-H (inner vs outer, high-density vs low-density) with different elastic properties has been well established in the literature. ${ }^{2,3}$ The locally dense and crystalline region we observe close to the cement grain surfaces with the LS interaction could therefore mirror what is seen experimentally, as does the fibrillar form taken far from the surfaces - demonstrating how these features can arise and persist as the interactions change during cement hydration. 


\section{Full Pore Size Distribution}

This section includes all plots of PSD at various $\phi$ for both ES and LS. Fig. S2 shows the pores near the system edge, or close to the cement grain surfaces. Examining the edge distributions, ES and LS seem to develop similarly with increasing $\phi$, though LS is more strongly varying. As $\phi$ increases and more particles are inserted, the pores at the edge grow smaller. LS has almost no porosity at high $\phi$, where we see pore size $s<1 \mathrm{~nm}$ - arising from the spacing between spheres in a crystalline packing. On the other hand, the ES pores get a bit smaller, but are still maintained through $\phi=25 \%$. This ability of ES to maintain porosity could be an important feature in cement hydration: allowing diffusion of water and ions between the pore solution and cement grain surface.
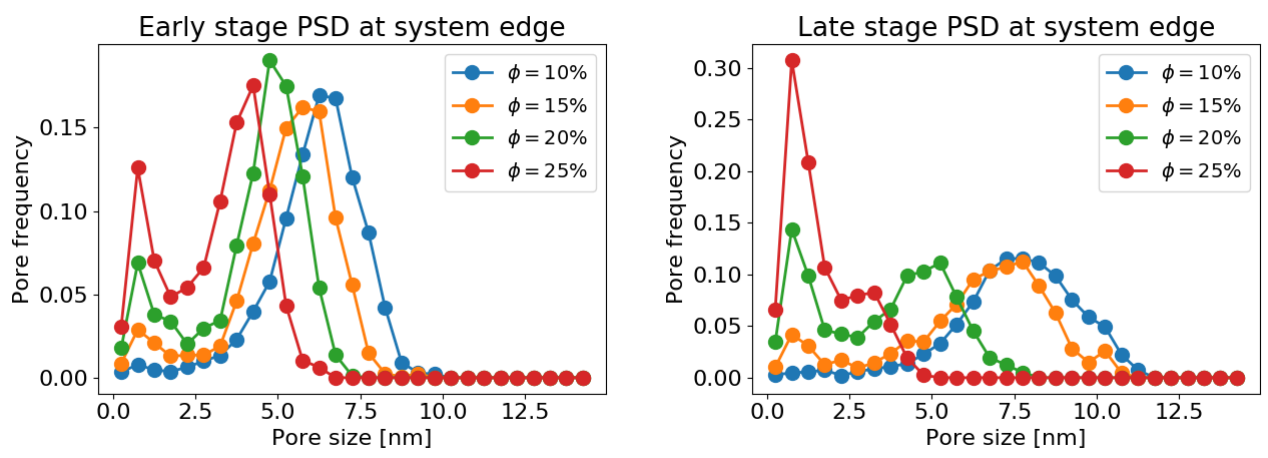

Figure S2: Plots of pore size distributions at the system edge, close to cement grain surfaces. The large decrease in pore size for LS corresponds to a large increase in local density.

PSDs from the center region of our system (Fig. S3) highlight the importance of the percolation for both potentials. Both ES and LS porosity in the center region are strongly affected by percolation. Before $\phi=15 \%$ both ES and LS have shrinking pores, as in the edge distributions. For ES, the percolation transition sets the characteristic pore size, and subsequent increase in density widens the distribution but does not affect the mean size. For LS, the percolation really signals a large deviation between the edge and center. While the edge porosity shrank towards non-existence, the center porosity coarsens and shifts to increasingly larger pores. This is consistent with coarsening towards phase separation. 

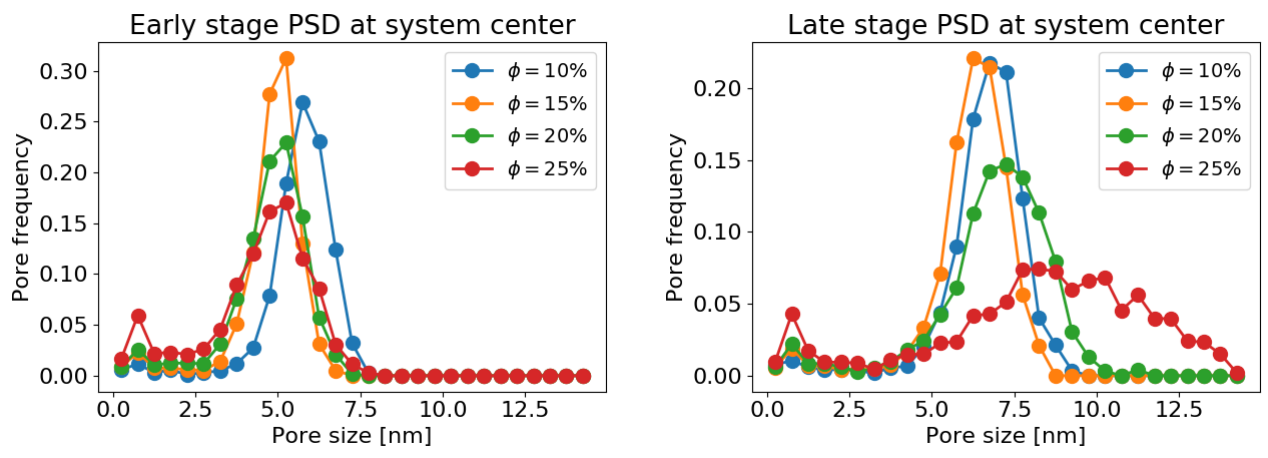

Figure S3: Plots of pore size distributions at the system center, far from cement grain surfaces. The large increase in pore size for LS is consistent with the start of phase separation through coarsening.

\section{References}

(1) Steinhardt, P. J.; Nelson, D. R.; Ronchetti, M. Bond-orientational order in liquids and glasses. Phys. Rev. B 1983, 28, 784-805.

(2) Tennis, P. D.; Jennings, H. M. Model for two types of calcium silicate hydrate in the microstructure of Portland cement pastes. Cem. and Conc. Res. 2000, 30, 855-863.

(3) Constantinides, G.; Ulm, F. J. The effect of two types of C-S-H on the elasticity of cement-based materials: Results from nanoindentation and micromechanical modeling. Cem. and Conc. Res. 2004, 34, 67-80. 